\title{
Navigation Dialog of Blind People: Recovery from Getting Lost
}

\author{
Jan Vystrcil, Ivo Maly, Jan Balata and Zdenek Mikovec \\ Czech Technical University in Prague, Faculty Of Electrical Engineering \\ Karlovo nam. 13, 12135 Praha 2 \\ Czech Republic \\ \{vystrjan, malyil, balatjan, xmikovec\}efel.cvut.cz
}

\begin{abstract}
Navigation of blind people is different from the navigation of sighted people and there is also difference when the blind person is recovering from getting lost. In this paper we focus on qualitative analysis of dialogs between lost blind person and navigator, which is done through the mobile phone. The research was done in two outdoor and one indoor location. The analysis revealed several areas where the dialog model must focus on detailed information, like evaluation of instructions provided by blind person and his/her ability to reliably locate navigation points.
\end{abstract}

\section{Introduction}

When blind people travel independently, it may happen that they get lost. This happens when they can not find any useful navigation points. Use of existing GPS based navigation systems is of no use as the maps do not provide appropriate navigation points and the GPS localization is imprecise (tens of meters, where the lost blind person needs precision at highest in meters). In such situation blind people typically use one of two following methods to recover. First they can ask people in their surrounding for help. Second they can call friend or dedicated assistance center. The first method is currently more favorable for blind people, but they have experience with both methods. In each method the dialog has different structure due to the different context information available to the helping person (called navigator) and lost blind person.

In our research we focus on the second method, navigation through mobile phone call. Balata et al. (2013b) showed that such method is usable and navigator can successfully guide blind person in outdoor environment. This is because the blind person is able to efficiently describe his/her position. Balata et al. (2013a) found that there is quite good coverage of locations that are very well known to blind persons and that they should be able to navigate other lost blind person there.

These findings show that building some kind of assistance center where blind people can help each other is a promising idea. Our intention is to extend such a center in a way that the helping person will be replaced by natural language based dialog system. According to Pittermann (2005) this dialog system belongs to the category "Dialog as purposeful activity" with overlapping to the category "Dialogue as collaborative activity". The key questions we focus on are:

- How the selection of an appropriate form of language depends on aspects of the environment?

- What is the structure of the dialog with respect to the environment?

In the initial step of this work-in-progress research, we want to analyze the communication between lost blind person and the navigator in order to analyze the dialog structure and make initial observation about the context information interchange and verification dialog. Such dialog is very important for navigator to find out, where exactly the blind person get lost. With the knowledge of the way of communication between lost blind person and navigator we will be able in the future replace the navigator with a navigation system based on natural language understanding.

In order to gather and analyze initial data we ran an experiment in which the blind person got lost and was asked to call the assistance center which mediated connection to suitable navigator. Together, they tried to find the actual position of blind person and they tried to navigate him/her to end of the track. 


\section{Related Work}

Many current dialog systems are based on statistical approach when analyzing the semantics of spoken dialog as presented by Jurcicek (2007). Using belief state tracking provides better results for cases of noisy input. Ma et al. (2012) introduced system that is combining geographical knowledge of landmarks with dialog system itself and work with probabilities of particular locations.

Recovering from lost scenario can be also compared to robot localization problem as presented by Thrun (1998) and Thrun et al. (2001), more exactly to kidnapped robot problem, where robot with knowledge of its position is moved to different location without providing this information to the robot. This scenario is testing the ability of robot to recover from being lost while expecting to be on another place. These methods are based on probabilistic algorithms, working with probabilities of measurement while being on a certain place.

However we do not expect blind person to wear any precise sensors for distance measurements and localization, we can benefit from his/her senses (touch, hearing and olfaction) that can provide set of reliable observations.

\section{Experiment Description}

\subsection{Collected Data}

We set up an experiment in order to collect and analyze initial data about the dialog structure of lost blind person and sighted navigator person. During the experiment we recorded the course of the test with two cameras, one was on the blind person's shoulder and one was used for 3rd person view of the scene in order to show context (environment) of the test. Moreover, we recorded the blind person's position using GPS coordinates in outdoor and blind person's interaction with mobile navigation application. Camera recordings and GPS logs were used only for post-test evaluation. The dialog between the blind person and navigator was recorded and annotated.

\subsection{Participants}

For the experiment, 13 blind people, 8 female and 5 male, were invited by e-mail and following snowball effect. All the participants had blindness of category 4 and 5 - according to ICD-10 WHO classification.

\subsection{Procedure}

In the experiment, we focused on three types of location, two outdoor and one indoor: city center streets (track A), open city park (track B) and university building (track $\mathrm{C}$ ). We selected these three types of location in order to analyze possible differences in the dialog structure or types of provided information.

The script of the experiment was similar for each type of location. The participant was given a mobile phone with mobile navigation application for blind called NaviTerier Vystrcil et al. (2012). NaviTerier provides TTS synthesized description of the predefined track divided into segments. For each segment the description of the environment and navigation to the next segment was tailored with respect to the way how blind people navigate. Borders of segments are selected on places that could be easily recognizable by blind people (e.g. corner of building, doors, etc.). Each participant had a goal to go from start point to the end of the track using the mobile navigation application for blind. In order to put the participant into the "recovery from lost" situation, the navigation instructions were intentionally modified to represent a mistake in the track description (a realistic mistake), which caused that the participant get lost. When the participant realized that he/she is lost, a navigator from assistance center was called and they tried to find out the location of blind person and navigate him/her to the end of the track.

Navigator was seated in an office without visual contact to lost blind person. He knew all three routes very well. The only source of information about the lost blind person was dialog done by a phone call.

\subsubsection{Track A - City Center Streets}

In track A the participant was asked to navigate to the Vaclavska passage, see Figure 1. The navigation instruction were changed so that the two streets (Trojanova and Jenstejnska) were switched so that the participant get lost at the $\mathrm{T}$ crossing of Jenstejnska and Vaclavska street. The navigation using the mobile navigation application for blind in this type of environment was easy for participants and they all get lost at the desired location.

The navigator and participant had several navigation points there to get oriented. First of all, there was a nearby busy street Resslova, which can be heard. Next there was a closed gateway with 
metal gate, which is quite unique for this location. There were also waste bins (containers), phone booth and entrance to the underground garage.

\subsubsection{Track B - Open City Park}

In track B the participant was asked to navigate through the park to the restaurant, see Figure 1. The navigation instruction were changed so that the two junctions were skipped and the participant ended near the middle of the park, where fountain is located.

In this type of location, there were also not many unique navigation points. The most usable were two perpendicular streets with trams, the fountain in the middle of the park and two unique stairs. There were also multiple benches and grass plots.

\subsubsection{Track C - Building}

In track $\mathrm{C}$ the participant was asked to navigate through the building from the entrance to the yard, see Figure 1. The navigation instructions were changed so that instead of taking stairs down, the participant was asked to take stairs up and he/she got lost in the small corridor, where the last doors should be located but they were not there. The navigation using the NaviTerier application in this type of environment was easy for the participants and they all get lost at the desired location.

At the place, where the participant got lost, there were several navigation points. First point was showcase from metal and glass at the expected location of doors to the yard. Then there was wooden door secured with metal bars and wooden stairs going up and down.

\section{Results and Discussion}

In the track $\mathrm{A}$ and track $\mathrm{C}$, the participants got lost at location very well known to the navigator, thus the identification of lost blind person location was mostly fast and easy. In the track B, participants got sometimes lost at locations unfamiliar for the navigator due to the ambiguity of the environment and thus the location identification process was complicated.

The dialog structure of the communication between lost blind person and the navigator corresponds to the model introduced by Balata et al. (2013b). At the beginning the blind person describes his/her location, track instructions and the problem description, i.e. what is the difference between instructions of navigation application and

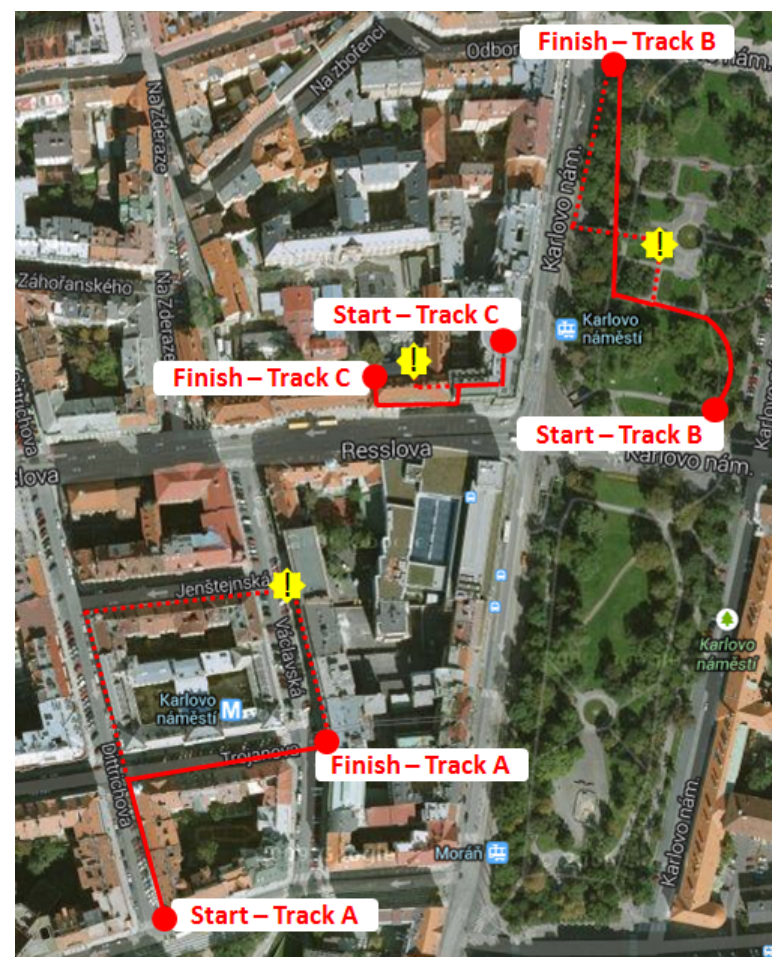

Figure 1: Visualization of individual tracks A, B and $\mathrm{C}$ used in the experiment. The intended path is shown by solid line. Path shown by dashed line shows the real path leading to the point, where the participant got lost - yellow exclamation mark and from where the participant was navigated back to the path.

reality. After the beginning the dialog continues by iterative searching of unique navigation points that may help the navigator to find the position and orientation of the lost blind person, until he/she gets to the location from which he/she can continue with the track. The dialog system should take into account following findings about the dialog structure.

When the blind person get lost, he/she uses information, provided by navigation application for sections that seemed to him/her correct and corresponding with reality, for description of his/her current position, e.g. "I am in the Vaclavska street." The dialog system should take into account uncertainty of information provided by lost blind person, possibility that the blind person got lost much earlier and the navigation instructions for next several segments were corresponding with the reality by coincidence.

The fact that the blind person gets lost is little bit stressy for him/her. Therefor he/she may provide illogical answers to some questions, e.g. 
Q: "Could you provide me with the description of your current position?" and A: "I would rather go to the start of the track and describe the track from the beginning."

Description of current position of blind person is very different from the description of sighted person. The dialog system should take into account that the blind person may not find particular navigation point, but it does not mean that the navigation point is not there. Moreover, some navigation points may be difficult or impossible to find by blind person. Similar issue is identification of particular navigation points. The blind person may have difficulties to distinguish between bend, turning, intersection and end of pavement. This may be misleading to dialog system. On the other hand, when the blind person confirms that particular navigation point was found, the system should check, if it is really the one, e.g. when doors are found, the system should check the material or type of the doors.

Blind persons use other senses than sight to scan the current position and navigation points. Even though the senses are more sensitive, the provided information may not be accurate, e.g. the blind person is reporting inclining pavement and in reality there is flat pavement.

It seems that the preferred sense is connected with the type of environment. In the track B with low density of navigation points which are ambiguous the blind persons preferred hearing.

Some navigation points are not permanent and may by varying. E.g. when there are two streets, one near (not busy) and one far (busy) and the blind person is asked to locate busy street, this information will depend on the current traffic on both streets. Together with the fact that term busy is subjective, the blind person may locate wrong street.

Some blind persons (the ones with high confidence of independency and orientation skills) tended to get oriented independently to the dialog with navigator. That means they provided the navigator with required information, but at the same time they were moving and they were disrupting the navigators mental model of the blind person's location.

There is not a standardized vocabulary how blind persons describe objects. Therefore they tend to use wide range of words and also metaphoric descriptions to describe the same ob- ject.

\section{Conclusion and Future Work}

In this paper, we did initial analysis of dialogs between blind person, who got lost when walking on a track with the instructions from mobile navigation application, and navigator, who is trying to help him get oriented. The research was done in three different locations, in city center streets, in open city park and in building. The dialog between blind person and navigator was recorded and qualitatively analyzed in order to reveal dialog features which can be used for improvement of the navigation itself and later it can help to replace the human navigator with automated system.

Initial analysis showed that the type of location may have impact on strategy, how the blind person explore his/her surroundings and how he/she tries to get oriented. In city center streets (track A) and in building (track C) the blind persons were able to explore their surroundings and they allowed the navigator to find out, where they probably are. In open city park (track B) the blind persons had problem to find navigation points and sometimes they were trying to get oriented independently, which led to the difficulties for navigator to find their position. In many cases, the blind persons were using the information from mobile navigation application until the point where they got lost. Unfortunately, such information may already be misleading. As a general finding, the dia$\log$ should focus also on verification of navigation points, which may not be permanent (e.g. finding busy street, when there are more streets around) or which may be not identified in not enough detail.

In future, we would like to focus on individual aspects found in qualitative analysis and design strategies into the dialog model between lost blind person and navigator and evaluate it quantitatively.

\section{Acknowledgments}

This research has been supported by the project Design of special user interfaces funded by grant no. SGS13/213/OHK3/3T/13 (FIS 161 832130C000).

\section{References}

J. Balata, J. Franc, Z. Mikovec, and P. Slavik. 2013a. Collaborative navigation of visually impaired. Journal on Multimodal User Interfaces, pages 1-11. 
J. Balata, Z. Mikovec, and J. Novacek. 2013b. Field study: How Blind People Communicate While Recovering From Loss of Orientation. In Cognitive Infocommunications (CogInfoCom), 2013 IEEE 4th International Conference on, pages 313-317, Budapest. IEEE Hungary Section, University Obuda.

Filip Jurcicek. 2007. Statistical approach to the semantic analysis of spoken dialogues.

Yi Ma, Antoine Raux, Deepak Ramachandran, and Rakesh Gupta. 2012. Landmark-based location belief tracking in a spoken dialog system. In Proceedings of the 13th Annual Meeting of the Special Interest Group on Discourse and Dialogue, SIGDIAL '12, pages 169-178, Stroudsburg, PA, USA. Association for Computational Linguistics.

Johannes Pittermann. 2005. Spoken dialogue technology: Toward the conversational user interface by michael f. mctear. Comput. Linguist., 31(3):403406, September.

Sebastian Thrun, Dieter Fox, Wolfram Burgard, and Frank Dellaert. 2001. Robust monte carlo localization for mobile robots. Artificial Intelligence, 128(12):99 - 141.

Sebastian Thrun. 1998. Bayesian landmark learning for mobile robot localization. Machine Learning, 33(1):41-76.

J. Vystrcil, Z. Mikovec, and P. Slavik. 2012. Naviterier - indoor navigation system for visually impaired. In SMART HOMES 2012, pages 25-28. Czech Technical University. 